\title{
PARTICIPATORY AND INCLUSIVE ASSISTIVE TECHNOLOGY INNOVATION CLINICS IN DESIGN SCHOOLS
}

\author{
Bhatnagar, Tigmanshu (1); Patel, Rohit (2); Roopchandani, Bhavik (3); Ashraf, Faisal (4)
}

1: University College London; 2: Indian Institute of Technology Delhi; 3: National Institute of Technology Kurukshetra; 4: v-shesh Access Services Private Limited

\begin{abstract}
People living with disabilities can have needs for Assistive Technology (AT) that are out of the scope of occupational therapists, commercial markets and charitable distributions. For such needs, designers, engineers, makers and clinicians in the local community can design and fabricate AT through an inclusive, participatory, user centred design process. By tapping into the skills, creativity, facilities and knowledge of local design, medical, engineering and management schools, we can make clinics for AT innovation, practical design education, business incubation and product provision. Through two case studies, we demonstrate the necessary steps towards this novel approach to compassionately design, fabricate and deliver bespoke and scalable AT innovations. The practice is multidisciplinary, it empowers people with disabilities to creatively challenge their problems, contributes to design education and requires a system to ensure product quality and follow ups. We envision that over years, this practice can become a movement that is able to systematically knit the patched ecosystem for AT, while contributing to the global understanding of design for people with disabilities.
\end{abstract}

Keywords: Inclusive design, Design process, Participatory design, Innovation

\section{Contact:}

Bhatnagar, Tigmanshu

University College London

University College London Interaction Centre

United Kingdom

t.bhatnagar.18@ucl.ac.uk

Cite this article: Bhatnagar, T., Patel, R., Roopchandani, B., Ashraf, F. (2019) 'Participatory and Inclusive Assistive Technology Innovation Clinics in Design Schools', in Proceedings of the 22nd International Conference on Engineering Design (ICED19), Delft, The Netherlands, 5-8 August 2019. DOI:10.1017/dsi.2019.403 


\section{INTRODUCTION}

Due to lack of affordable and appropriate product options and diminished means to address needs from a policy point of view, the issue of providing appropriate Assistive Technology (AT) to people with disabilities has become a wicked problem (de Witte et al., 2018). Universally designed, mass manufactured and mass distributed AT does not always fit people's needs, which in turn leads to high rates of abandonment (Philips and Zhao, 1993). Occupational therapists and rehabilitation engineers tailor, craft and repurpose objects to suit the needs of their patients (De Covereur and Goossens, 2011) but these solutions are bespoke and do not permeate the larger market space. With the advent of digital manufacturing, new possibilities have emerged such as the e-nable prosthetic hand, which allows for personalised, inexpensive and repairable AT (Hofmann et al., 2016). However, often, and especially in low and middle income countries, there is a lack of both trained healthcare professionals and local fabrication knowledge and facilities, which stifles the availability of AT for people. Therefore, families and local communities fabricate ATs for themselves, making use of what they have and ingeniously crafting material artefacts to achieve the assistance that they can (Werner et al., 1998). The global picture of AT innovation therefore, looks more of a patchwork than a systematic ecosystem.

This patchwork, presents opportunities. There is a clear need for AT innovation that is affordable, adaptable, available and much of the need requires bespoke manufacturing and craft which, given the rise of digital manufacturing methods provides a considerable opportunity for new entrepreneurial activity. Designers, engineers, makers, clinicians along with people with disabilities in their local community can try to address this gap by working together. In this paper, we demonstrate the initial steps towards a novel approach for a possible way to design, fabricate and deliver AT by tapping into the skills, creativity, facilities and knowledge available in local design, medical, engineering and management schools, making them clinics for AT innovation, practical education, fabrication and provision.

In such academic settings, innovative ideas can incubate in a safe, creative and fostering environment. They are shielded from the pressures of economics, guided by academic expertise and are encouraged to be innovative, user focussed and impact driven towards the betterment of the local environment. Such hubs will be instrumental in producing the necessary research to enhance our collective understanding of the lives, needs and solutions for people who require AT, which at this moment, of meagre quantity and quality (Borg et al., 2011). Over the years, we expect that such an approach will help many people with disabilities, train students through practical user centric design projects and evolve to create a local impact that can produce globally relevant solutions.

In this paper, we reflect on two projects undertaken at the Department of Design, Indian Institute of Technology Delhi. These projects are described in Section 2. In Section 3, we highlight four key experiential learnings from the cases, three of which we argue are necessary for effective AT innovation and one which makes the practice meaningful in an academic setting.

\section{PROJECT CASES}

\subsection{Fulfilling the needs for personalised and complex AT}

Madhav (name changed), a 22-year-old young man has been living with spastic quadriplegia for the past 20 years due to a complication that occurred in surgery when he was a child. Ever since, he has been dependent on his parents for his activities of daily living and social participation. His family cannot afford expensive AT such as a powered wheelchair or a full time carer. They face difficulties to balance between the level of care and support that their son requires and their professional responsibilities. As they are getting older, their physical capabilities are dwindling, they have started to present medical issues and were looking for something that can help.

Multiple engagements with the user and his family were made by a design academic, an engineering student, a maker and a physiotherapist. This led to enhanced understanding of the needs of the family from different perspectives and allowed for a collaborative partnership to develop to address the family's needs. Some of the engagements were in the context of their home, where they could show, tell and demonstrate how a day in their life is. Semi-structured interviews were conducted in such 
settings to understand the context and the rationale behind their adapted activities, in order to discuss ideas and evaluate potential design interventions. It was strongly remarked by Madhav's parents that being facilitated to think of bringing a change was empowering. The team collaboratively developed an idea for a device that could help the Madhav stand, so it gives him a psychological boost, stretches his contracted joints, corrects his posture, provides load to the muscles in his limbs and helps his parents to transfer him from place to place in the house. As the device would need to be mainly operated by the parents, it was essential that it is easy to use, robust, compact and requires as little maintenance as possible.

The Basic Design Cycle (Roozenburg and Eekels, 1995) was followed to conceptualise the idea which quickly moved to prototyping. The motion of sit to stand was achieved by a four bar crank rocker mechanism (Dynref.engr.illinois.edu, 2019) actuated by an off the shelf hydraulic jack. Once the team built the first prototype, the family was invited to the academic makerspace to evaluate the design. The evaluation was conducted under the supervision of two physiotherapists who offered a clinical critique to the functionality and causes of potential harm from the device. The feedback from the users, medical experts and other makers and designers was taken into account through think-aloud discussions while Madhav and his parents used the device. The entire event was video recorded, which was then used to further develop the product. Based on the feedback, the design was refined by making it lighter, more robust, secure and comfortable and appropriate for Madhav's physical measurements and fabricated again using industrial manufacturing techniques available at the institutional makerspace. The family, the user and clinical experts were again invited to the workshop to evaluate the product and provided feedback on finer details. After the suggestions were incorporated, Madhav began using the device. His parents have been recording his activities over time under the guidance of the physiotherapist and have access to on-demand device related technical support by the engineering student and the technician involved in the fabrication of the device.
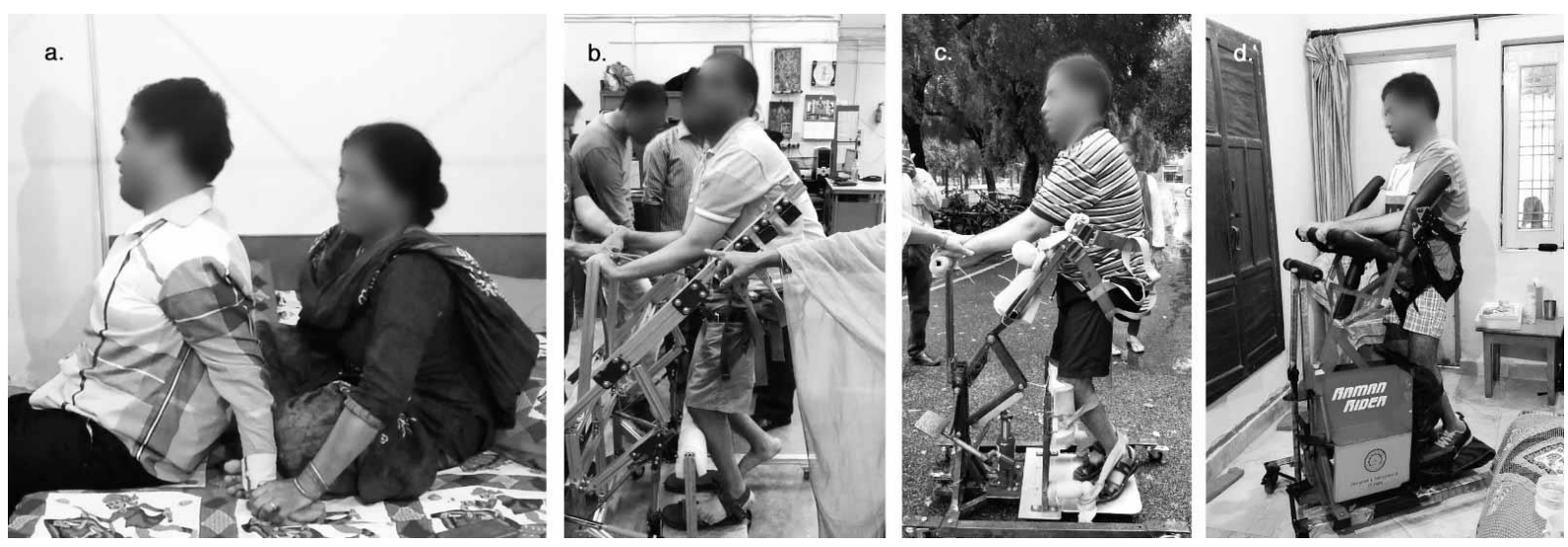

Figure 1: (a) Madhav's parents demonstrating various effects they have observed over the years. (b) Madhav evaluating the first prototype device to help him stand up under the supervision of physiotherapists, his parents, other designers and makers (c) Evaluation of the second prototype, the user and the family had the confidence to make him stand straight up and move around. This picture was taken outside the building. (d) Madhav using the final product at home.

\subsection{Supporting Inclusive Product / AT enterprise}

Saif (name changed) is 28 -year-old, has a congenital lower limb abnormality and uses a wheelchair. He had his motorcycle adapted for daily commute. The adaptation was done by a local mechanic who provides modification services of motorised vehicles for people using wheelchairs, crutches and lower limb braces. The user was highly dissatisfied with how the motorcycle operated after the modification and had previously met with an accident due to a mechanical failure in the modification. "Being the only form of design adaptation available, the product is highly stigmatised with little care to aesthetics and engineering optimisation" said Saif. Seeing no better option available in the market, he was motivated to take the challenge in his own hands and make something that is dignified, safe and functional for himself, with a possibility to build an enterprise in future as he knew of many other wheelchair users who are faced with similar challenge on a daily basis. 
Through a series of meetings among the user, design academic, engineering, design and management students, a rich picture of the Saif's requirements and a sense of mutual trust was built. The existing system was analysed and the state of the art for such products was studied. A survey was conducted with over 150 people with mobility disorders from various cities in India. It reported that over $92 \%$ of the people who are using such a modification feel that there can be a better adaptation to their two wheelers compared to the ones that are currently available. The information from the survey and multiple brainstorming sessions - building on the experiential knowledge of the user and his friends and guided by the skills and expertise of the engineering, design and management student's team - led to a desirable, functional and economically feasible concept of a tilting reverse trike.

Reverse Trike is mechanical configuration of motorised vehicles that has two wheels in front and one at back. It is stable at zero speed compared to two wheelers and offers better cornering capabilities than three wheelers with two wheels at the back (delta) (Endeavor Trikes, 2019). At the same time, it's more dynamic and offers better manoeuvrability than a four-wheel diamond pattern currently being adopted as a retrofit modification for people with disabilities. As the user had never experienced riding a reverse trike, a prototype was developed frugally. An old, motorcycle was repurposed with a custom built frame and second hand vehicle components. Specific components were acquired from local hardware stores and the engineering details were adapted to the available components. The prototype was first evaluated by the makers for its safety and robustness and then it was trialled by the user in controlled conditions. The feedback of the user provided new hints on how a person without lower limb control, sensation or presence would like to ride three wheeled motorcycles. This aspect is a topic for further research.

Another motorcycle was hacked and fabricated using industrial manufacturing facilities at the institutional makerspace and workshop. Based on the experiential understanding based on the feedback of the user and other participants, the design was refined. Engineering students and makers were heavily involved in the development of a proof of concept prototype. They were complimented by design students who developed the aesthetical and usability concepts of the vehicle. The efforts were further directed by a group of management students who simultaneously developed a business model through the SWOT method for the inclusive product in the Indian automotive market. Multiple users, both disabled and non-disabled, evaluated the prototype motorcycle and gave their subjective feedback on stability, balance, control and ride experience. "Clearly, this adaptation is proving to be better and more dignified than before. There are still some technical improvements necessary before you go to the market but coming this far is already a great achievement", said one of the participant. Another participant critiqued that "Riding this vehicle is completely different from a two wheeler. There is a significant learning curve for me, especially at turning I feel under-confident."
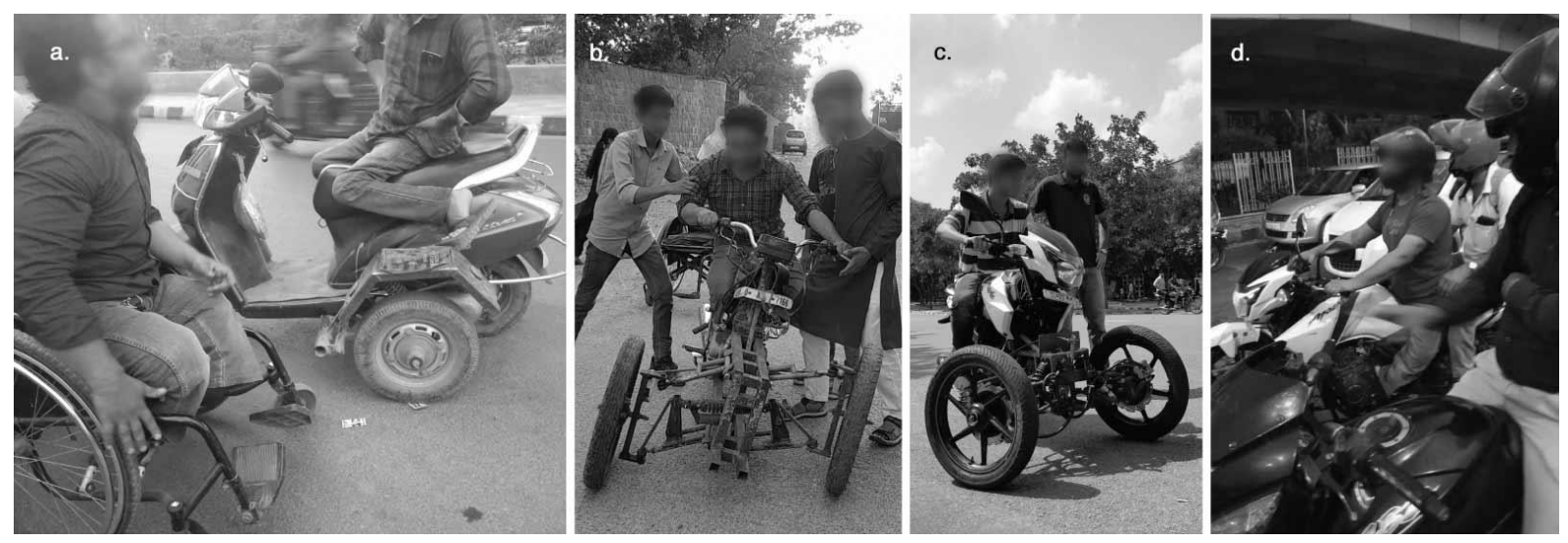

Figure 2: (a) Initial in-context user research with people using modified two wheelers. (b) User testing the primitive tilting reverse trike prototype. (c) Second improved prototype being tested by an able bodied user for subjective feedback on ride quality, dynamics, braking and control. (d) User testing the prototype bike on real street conditions.

\section{THE PROCESS}

Although the two cases are distinct versions of product design and development, in both the need for the project was initiated by the users. This made users engage actively with the team and critical about 
the results. It led to an obvious and acceptable product which they were proud of due to their involvement, ideas and inputs. The common people in the two cases were the makers and the academic who facilitated the two projects. Other than them, according to the needs of the user, a multidisciplinary team was formulated made of interested students and necessary subject experts. In both cases, multiple co-creation sessions were conducted in the respective contexts leading to the conceptual idea. It was followed by iterative design cycles until either the final product was ready to be delivered (in the first case) or a prototype was fabricated to be evaluated by other users (in the second case). Although depended on the skills and knowledge of the people involved, the process can ensure that the products are appropriately designed. For products that are made for bespoke needs, a system for follow up, longitudinal research for understanding the condition and/or adapting the design of the product to the changing needs of the user is necessary. We are currently working on the details of this system and hence, it is out of the scope of this paper. For the second type, a typical process for industrialisation of designed products, which is well documented in literature needs to be followed.

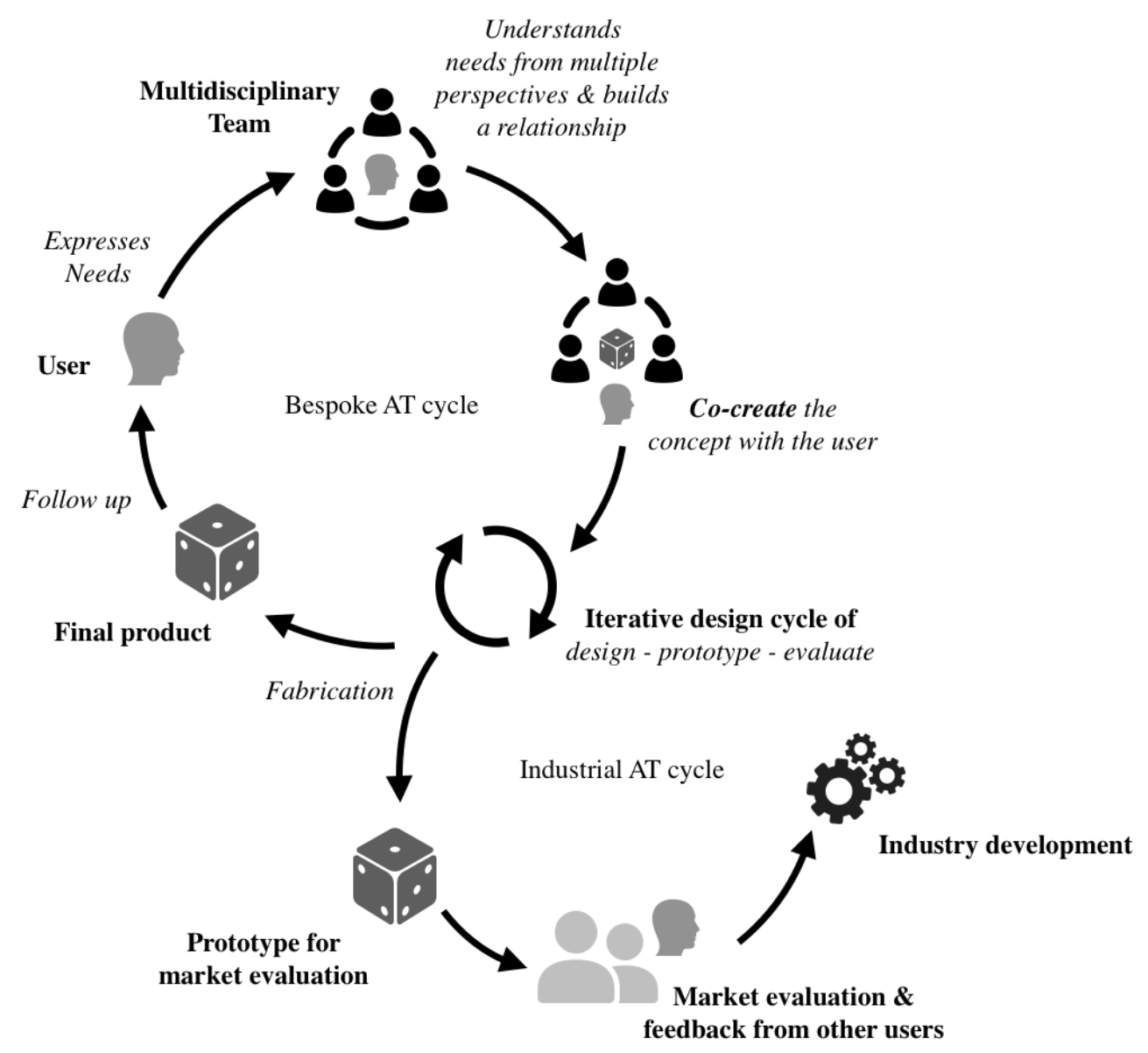

Figure 3: Schematic representation of the process in the two cases. The Bespoke AT cycle is derived from the first case and the Industrial AT cycle from the second. Both have the first steps in common but diverge on the intent of the final prototype / product of the design process.

\section{KEY LEARNINGS}

In literature, there is no definitive consensus about the most efficient design method for disabled and the factors that should be taken into account for choosing a method (Magnier et al., 2012). User Centred Design tools are widely used, often intuitively, although their implementation is reported to be difficult with disabled participants and their effectiveness is not yet established (Barbareschi, 2018). Tools and methods for the design of Assistive Technology are borrowed from the fields of Psychology, Rehabilitation Engineering, Psychophysics, Business Development on a case to case basis, generalisation of which is difficult. From the above two cases, we derive some common threads that are described below. 


\subsection{AT innovation is multidisciplinary}

There is an increasing repository of AT design ideas available in virtual communities that has been mainly generated by hobbyists with an interest in engineering (Buehler et al., 2015). Online sharing, access to internet, 3D modelling software and video instructions to fabricate and assemble promotes virtual collaboration (Buehler et al., 2015). Do it yourself AT can improve user's life by providing cost effective personalisation of devices eventually leading to reduced abandonment (Hurst and Tobias, 2011). However, few of these designers have clinical expertise or a direct experience of disability (Hofmann et al., 2016). Furthermore, there are instances when the needs of people with disabilities goes beyond what they can design and develop by themselves or what can be easily found over the internet. The provision of devices which could then fulfil their needs requires diverse expertise of designers, researcher, engineers, makers and clinicians (at times, business developers, psychologist and anthropologists) to work with people with disabilities.

Designers facilitate user's expression and lead to ideas which can achieve their desired experiences through material instantiations. By immersing themselves in the user's environment and generating empathy for the user's life, researchers can develop a better understanding of the problem that needs to be solved. Through collaborative ideation exercises, a notion of what is needed and how it can be achieved begins to emerge in the form of statements such as, "It would be great if there is something that does...". For most cases of AT design, these conversations should be in the presence of medical experts to avoid unforeseen harm to the user and support interventions from a clinical perspective. The clinical understanding of the condition the user is facing and what would be beneficial for one's physical and cognitive maintenance or development over time, is at par with the desired experience the user wants to have in life. The holistic concept which emerges from this co creation needs to be detailed for fabrication, which requires engineering expertise. The technical specifications are then taken over by makers in order to fabricate the product. This entire exercise is iterative in nature; with concepts, technical specifications and eventually the product evolving through evaluations by the users, the designers, the engineers and the clinicians at multiple stages. At times, such inventions can be orphan and are made specifically for a single person. At times, they have to be produced in mass to be made available for many. Therefore, these activities emerge to become a kind of design consultancy, design education and a service provisioning system based on multidisciplinary collaboration.

\subsection{Participatory design empowers people with disabilities and their families}

People are experts of their own experience (Visser et al., 2005). Over the years of living with impairments, AT users have adapted to become skilled and practical creative problem solvers (Shah and Robinson, 2007) and they want to be involved in the development of products and services that they will use. Hurst and Kane (2011) argue that it is possible to build personalised AT, and empowering users to make their own Assistive Technology to improve the adoption of AT, thereby reducing abandonment.

Designers need to let users and other stakeholders enter their expertise 'solution space' and express themselves. Their intuition is quintessential for appropriate design. Several methods, or techniques, can support practice for enhancing the quality of participation have been documented in literature (Buur and Larsen, 2010), (Kettley et al., 2015). We observed that the participatory design process fuels the attitude to bring a change, which empowers people to identify their problems and address them instead of accepting. Users are also motivated by the team's dedication and passion towards problem solving. Visiting user on multiple occasions in their own environment gives a rich picture to designers, researchers and clinicians for more appropriate solutions. It also builds trust which makes users comfortable to express themselves, collaborate and become part of the team.

Meaningful participatory design requires prolonged engagement but participation can be burdensome for AT users (Barbareschi, 2018). Adding to this, the complex nature of disabling conditions and the variation it exhibits across individuals and environments - it can be appropriate to begin the design process by focussing on a single use case. This is because the undiluted involvement attributes an active, creative and decisive role to participants which makes them naturally motivated and engaged. This is counterintuitive as universal understanding and widely useful solution requires sufficient sample size of participants depending on the research question. However, people with disabilities are 
strongly connected in social groups (Moussaid et al., 2013). Hence, as understanding of the problem and solutions emerge, this network of users can be involved in the process to provide further feedback and guide the iterations.

Furthermore, with the understanding of product development process and co-creation, there is a tendency for the users to come about relevant latent information which can be of high value for the product development. For instance, while brainstorming on ways to correct the deformity of the hand in the first case, Madhav's mother demonstrated a practical phenomenon which she had observed over the years as to how her son's hand naturally improved grip at a particular posture. To this, the physiotherapist explained the anatomical phenomena and the requirement of a device that could maintain that posture was established, which could not have been anticipated.

\subsection{AT innovation and provisioning contributes to design education}

In an academic setting, AT design and innovation exercises can practically benefit the disabled community while providing important learning opportunities for students, (Barabareschi et al., 2017). These learning opportunities give students the experience to work parts of a real problem and directly see its impact on lives.

In the above projects, although students' direct involvement was for specific time and responsibilities in the assignment, they were motivated by the actual delivery of the product. The constraint of delivering a product, for which a user they know is waiting, adds a layer of responsibility to the work of the students. This practical sense of purpose can be a better motivator than academic grades or jury presentations, but requires close guidance by the academics. "The best part about the project was that we had to deliver the product to its user. Therefore, it was not abandoned until that goal was achieved which is not very common for us", said an engineering student involved in the projects. Another interning student commented, "Seeing the practical implication of my work and witnessing its impact was very good. I would not have experienced this in a company based internship."

We observed that, for the students, engaging with real users helps to develop a genuine understanding of the problem and a commitment to designing solutions. In the above projects, the involved students gained knowledge from a clinical, technical, social and business development point of view through both the advice of experts and practice. Furthermore, because of challenging nature of the assignments, an experimental but frugal mindset to design (Radjou and Prabhu, 2015), prototype and evaluate was encouraged, leveraging on the available facilities, access to expertise, materials and techniques.

The products being built are eventually used by people. Long term monitoring of the device and its effects on the condition can be done through the integration of sensors in the devices themselves or through charts in which data is manually inputted and maintained. It would be instrumental in improving the understanding of condition and to make the designs more relevant and user centered. As we encourage these projects to be conducted in the local contexts and in a participatory manner (Section 3.3), practical difficulties in prolonged engagement with users and longitudinal studies can be reduced.

Utilising facilities and expertise available in academic institutions, high quality of products and innovations can be achieved. These academic maker spaces are a safe heaven to experiment, innovate and build quality one-off products that can be given to people for desired utilisation, build prototypes that can support enterprise development and batch produce artefacts needed for experiments or trials. However, the act of design comes at the cost of time, material and money. People with disabilities are not always able to bear the cost of products and personnel developing them. They already suffer from economic discrimination (Mitra, 2006). Furthermore, the majority of people with disabilities who live in low and middle income countries cannot afford bespoke products (Rohwerder, 2018). Charity is not a sustainable approach for provision of AT (Smith et al., 2018).

Provision of the necessary AT to people with disabilities falls under the onus of the government under the UNCPRD. It's a complicated decision making process from the perspective of eligibility of support (de Witte et al., 2018). However, innovation of AT, especially through participatory design, has the potential to push the state of the art of science of design for the disabled. The development of products to enhance the capabilities of humans brings light to new research questions, which can only 
be explored while working with people with disabilities. To balance their participation in the contribution of science, it is justified that they receive the benefits of AT instantaneously. This pushes the boundaries of the design processes and leads to the development of new products which can be useful for many. Funds would also have to support potential spinoff innovations until they mature and are self sustainable. Therefore, the activities of AT innovation within academic makerspaces as described, should be initially supported by academic grants and governmental funding.

\subsection{Product quality and follow up needs to be ensured}

In some cases, custom products are fabricated for an individual for long term use. In some cases, more traditional industrial design and product development processes are employed where prototypes are constructed to be evaluated. At all occasions, for any form of user testing, the products / prototypes have to be validated and built with quality standards to avoid any form of harm. Measures to evaluate have been documented in literature and are widely used in industry such as FMEA and Human Error Analysis (National Research Council, 2007). These have to be adapted and applied to individual products which is produced and delivered and to prototypes meant for user evaluations. It is also recommended that user evaluations are done in the presence of clinical experts in order to safeguard the user from situations which could potentially harm the users.

People who design and build a particular product are best suited to address such needs but may not always available. Although, user involvement in the design stage helps them to be able to maintain safe workings of the device, it is advisable that the design is fabricated such that local mechanics can understand and preform minor repairs if necessary. For research purposes, users of the device need to be approached regularly to secure relevant data from a clinical and design point of view. Therefore, a system to provide follow-ups and maintenance is required. Although we provided on demand support, however a more systematic system needs to be in place.

If such a paradigm grows in quantity and product delivery becomes the prime challenge, new strategies to maintain quality and follow ups would have to be utilised, which are out of the scope at this moment.

\section{CONCLUSION}

We described two AT innovation projects done at an academic institute in India which lay the foundational thought for a Multidisciplinary AT Innovation Clinic in academic settings. Its primary focus is to provision bespoke AT and AT innovation support to local people with disabilities, opening new avenues to relevant research in AT innovation and design for the disabled, which is of a global value. Following are our key learnings from the activities:

- The process requires a collaborative and participatory involvement of a multi-disciplinary team of designers, researcher, engineers, makers and clinicians (at times, business developers, psychologist and anthropologists) to work with people with disabilities.

- Participation of people with disabilities and their families in the design process empowers them to overcome their difficulties rather than accepting them, inculcating a spirit to change for the better.

- $\quad$ AT innovation and provisioning practically benefits the disabled community while providing important learning opportunities for design, engineering, research, physiotherapy and management education.

- $\quad$ The fabricated product quality must meet standards and a system of follow-up is necessary for prolonged learning and design improvements.

We envision that over the years and batches of students, this act of 'Compassionate Creation' can become a movement that is able to systematically knit the patched ecosystem for AT needs, while contributing to the global understanding of design for disability.

\section{ACKNOWLEDGMENTS}

The authors would like to acknowledge the hard work and persistence of Mr. Prithviraj Vyas, Mr. Sanjay Kumar, Mr. Anil Kumar and Mr. Abhishek Sharma. We would also like to extend a heartfelt thanks to two young makers, Ayan Baig and Taha Wahaab for their never-ending energy and ingenuity. We would also like to thank members of the formula SAE team of IIT Delhi for their 
theoretical technical support and Anal Bangre for design inputs. A sincere thanks to Mr. and Mrs. Srivastava and Raman Srivastava for their generous support and for letting us in their lives. Enough acknowledgement cannot do justice to Mr. Faisal Ashraf, who guided us and taught us about disability beyond measure. He took risks, invested in our experiments and was immersed with us in the process. His feedback shaped the products and the relationship that is forged with him will extend for a very long time. We would also like to extend a great sense of respect and thank Dr. Shevta Katyal, Dr. Komal Kashyap and Dr. Shah Alam Khan for their immeasurable critical remarks and guidance. Without the continuous support and encouragement of Prof. PVM Rao of the Department of Design, from Prof. Naresh Bhatnagar and members of the Production Engineering Lab, IIT Delhi, none of this could have been achieved. Finally, a vote of thanks to the anonymous reviewers, Dr. Catherine Holloway and Dr. Giulia Barbareschi for their valuable feedback on the manuscript.

\section{REFERENCES}

Barbareschi, G., Holloway, C. and Sprigle, S.H. (2017, June), “Assistive Technology Design Courses: The Mutually Beneficial Relationship between Engineering Education and the Provision of Orphan Devices", http://www.resna.org/news-events/annual-meeting/annual-meeting-proceedings/annual-conferenceproceedings (Vol. 2017), Rehabilitation Engineering and Assistive Technology Society of North America (RESNA).

Barbareschi, G. (2018), YouTransfer, YouDesign: A participatory approach to design assistive technology for wheelchair transfers, Doctoral dissertation, UCL (University College London).

Borg, J., Lindström, A. and Larsson, S. (2011), "Assistive technology in developing countries: A review from the perspective of the convention on the rights of persons with disabilities", Prosthetics and Orthotics International, Vol. 35 No. 1, pp. 20-29. https://doi.org/10.1177/0309364610389351.

Buehler, E., Branham, S., Ali, A., Chang, J.J., Hofmann, M.K., Hurst, A. and Kane, S.K. (2015), "Sharing is Caring"“" Proceedings of the 33rd Annual ACM Conference on Human Factors in Computing Systems CHI 15. http://doi.org/10.1145/2702123.2702525

Buur, J. and Larsen, H. (2010), "The quality of conversations in participatory innovation", CoDesign, Vol. 6 No. 3, pp. 121-138. http://doi.org/10.1080/15710882.2010.533185.

De Couvreur, L. and Goossens, R. (2011), "Design for (every) one: co-creation as a bridge between universal design and rehabilitation engineering", CoDesign, Vol. 7 No. 2, pp. 107-121. https://doi.org/10.1080/15710882.2011.609890.

de Witte, L., Steel, E., Gupta, S., Ramos, V.D. and Roentgen, U. (2018), “Assistive technology provision: towards an international framework for assuring availability and accessibility of affordable high-quality assistive technology", Disability and Rehabilitation: Assistive Technology, pp. 1-6. https://doi.org/10.1080/17483107.2018.1470264.

Dynref.engr.illinois.edu. (2019). Four-Bar Linkages. [online] Available at: http://dynref.engr.illinois.edu/aml.html [Accessed 15 Mar. 2019].

Endeavor Trikes (2019). Why Reverse? - Endeavor Trikes. [online] Available at: https://endeavortrikes.com/why-reverse/ [Accessed 15 Mar. 2019].

Federal Policies and the Medical Device Industry (Washington, D. C.: U.S. Congress, Office of Technology Assessment, OTA-H-230, October 1984)

Hofmann, M., Burke, J., Pearlman, J., Fiedler, G., Hess, A., Schull, J. and Mankoff, J. (2016), "Clinical and Maker Perspectives on the Design of Assistive Technology with Rapid Prototyping Technologies", Proceedings of the 18th International ACM SIGACCESS Conference on Computers and Accessibility ASSETS 16. http://doi.org/10.1145/2982142.2982181

Hurst, A. and Tobias, J. (2011), "Empowering individuals with do-it-yourself assistive technology", The Proceedings of the 13th International ACM SIGACCESS Conference on Computers and Accessibility ASSETS 11. http://doi.org/10.1145/2049536.2049541

Kettley, S., Kettley, R. and Bates, M. (2015), "An introduction to IPR as a participatory design research method", Proceedings of the 2015 ACM International Joint Conference on Pervasive and Ubiquitous Computing and Proceedings of the 2015 ACM International Symposium on Wearable Computers UbiComp 15. http://doi.org/10.1145/2800835.2807944

Magnier, C., Thomann, G., Villeneuve, F. and Zwolinski, P. (2012), "Methods for designing assistive devices extracted from 16 case studies in the literature", International Journal on Interactive Design and Manufacturing (IJIDeM), Vol. 6 No. 2, pp. 93-100. http://doi.org/10.1007/s12008-012-0143-2.

Mitra, S. (2006), "The capability approach and disability", Journal of disability policy studies, Vol. 16 No. 4, pp. 236-247. http://dx.doi.org/10.2139/ssrn.2330438.

Moussaï, M., Kämmer, J.E., Analytis, P.P. and Neth, H. (2013), "Social influence and the collective dynamics of opinion formation", PloS one, Vol. 8 No. 11, pp. e78433. https://doi.org/10.1371/journal.pone.0078433. 
National Research Council (2007), Human-system integration in the system development process: A new look, National Academies Press.

Phillips, B. and Zhao, H. (1993), "Predictors of assistive technology abandonment", Assistive technology, Vol. 5 No. 1, pp. 36-45. https://doi.org/10.1080/10400435.1993.10132205.

Radjou, N. and Prabhu, J. (2015), "Frugal Innovation: How to do more with less", The Economist.

Rohwerder, B. (2018. Assistive technologies in developing countries.

Roozenburg, N.F. and Eekels, J. (1995), Product design: fundamentals and methods, Vol. 2, John Wiley \& Sons Inc.

Shah, S.G.S. and Robinson, I. (2007), "Benefits of and barriers to involving users in medical device technology development and evaluation", International journal of technology assessment in health care, Vol. 23 No. 1, pp. 131-137. https://doi.org/10.1017/S0266462307051677.

Smith, R.O., Scherer, M., Cooper, R., Bell, D., Hobbs, D.A., Pettersson, C., Seymour, N., Borg, J., Johnson, M.J., Lane, J.P. and Srinivasan, S.S. (2018), “Assistive technology products: a position paper from the first global research, innovation, and education on assistive technology (GREAT) summit", Disability and Rehabilitation: Assistive Technology, pp. 1-13. https://doi.org/10.1080/17483107.2018.1473895.

Visser, F.S., Stappers, P.J., Van der Lugt, R. and Sanders, E.B. (2005), “Contextmapping: experiences from practice”, CoDesign, Vol. 1 No. 2, pp. 119-149. https://doi.org/10.1080/15710880500135987.

Werner, D., Thuman, C. and Maxwell, J., 1998. Nothing about us without us. Developing innovative technologies for, by and with disabled persons. Palo Alto: Healthwrights. 